\title{
Prácticas de crianza parental y conducta antisocial en adolescentes en conflicto con la ley
}

Parenting practices and antisocial behavior in adolescents in conflict with the law

Práticas parentais e comportamento anti-social em adolescentes em conflito com a lei

\author{
Germán Cabrera Gutiérrez ${ }^{1}$ \\ Nora Helena Londoño Arredondo² \\ Joan Sebastián Arbeláez Caro ${ }^{3}$ \\ María Camila Álvarez Martínez ${ }^{4}$ \\ Andrés Mauricio Gamboa Hernández ${ }^{5}$
}

Recibido: 25 de agosto de 2020 Aprobado: 27 de octubre de 2021 Publicado: 3 de noviembre de 2021

Cómo citar este artículo: Cabrera Gutiérrez, G., Londoño Arredondo, N.H., Arbeláez Caro, J.S., Álvarez Martínez, M.C. y Gamboa Hernández, A.M. (2021). Prácticas de crianza parental y conducta antisocial en adolescentes en conflicto con la ley. Pensando Psicología, 17(1), 1-22.

doi: https://doi.org/10.16925/2382-3984.2021.01.03

Artículo de investigación. https://doi.org/10.16925/2382-3984.2021.01.03

1 Universidad de San Buenaventura Medellín, extensión Armenia.

Correo electrónico: german.cabrera@usbmed.edu.co

ORCID: https://orcid.org/0000-0001-9284-3467

2 Universidad de San Buenaventura Medellín.

ORCID: https://orcid.org/0000-0001-6016-1955

3 Universidad de San Buenaventura Medellín, extensión Armenia. ORCID: https://orcid.org/0000-0001-9935-5781

$4 \quad$ Universidad de San Buenaventura Medellín, extensión Armenia. ORCID: https://orcid.org/0000-0002-0702-5150

5 Universidad de San Buenaventura Medellín, extensión Armenia. ORCID: https://orcid.org/0000-0003-4182-4009 


\section{Resumen}

Objetivo: determinar la relación entre las prácticas de crianza parental y la conducta antisocial en adolescentes vinculados al sistema de responsabilidad penal para adolescentes.

Método: el presente estudio se enmarcó en una investigación cuantitativa transversal, descriptiva-correlacional. La población fueron los adolescentes vinculados al sistema de responsabilidad penal y la muestra se determinó siguiendo un muestreo no probabilístico-intencional. En total, participaron 70 adolescentes vinculados al sistema de responsabilidad penal para adolescentes (SRPA).

Instrumentos: ficha de caracterización, escala de prácticas parentales de Andrade y Betancourt (2008) y la entrevista neuropsiquiátrica para adolescentes (Ferrando et al., 2000).

Resultados: el $80 \%$ refiere trastorno de conducta antisocial. Se encontró, además, que el control conductual materno tiene diferencia significativa respecto al grupo de las personas con trastorno antisocial $(U=1134,500$; $p=0,014) y$, en el caso del grupo de género, la diferencia estaba en el control psicológico materno ( $U=354.000$; $p=0,00)$. El control conductual materno mostró relacionarse con el trastorno de conducta disocial (Rho $=$ $0,222 ; p=0,014 \leq 0,05)$. El control psicológico materno y el género se relacionaron $(\operatorname{Rho}=0,240 ; p=0,00 \leq 0,01)$. Conclusiones: la variable determinante para el caso de la muestra de adolescentes con conducta antisocial fue el control conductual materno y según el género fue el control psicológico, confirmando la importancia que tiene la influencia de las prácticas maternas en la construcción del comportamiento prosocial en adolescentes.

Palabras clave: conducta disocial, conducta del adolescente, prosocialidad, responsabilidad parental.

\section{Abstract}

Objective: Establish the relationship between parenting practices and antisocial behavior in adolescents linked to the criminal responsibility system for adolescents.

Method: The present study was framed in a quantitative, descriptive-correlational research of transversal temporality. The population was adolescents linked to the criminal responsibility system and the sample was determined following a non-probabilistic-intentional sampling. In total, 70 adolescents linked to the criminal responsibility system participated.

Instruments: Characterization sheet, scale of parental practices by Andrade and Betancourt (2008) and the neuropsychiatric interview for adolescents (Ferrando et al., 2000).

Results: $80 \%$ of the population reported antisocial behavior disorder, it was also found that maternal behavioral control has a significant difference from the group of people with antisocial disorder $(U=1134,500 ; p=.014)$ and in case of the gender group, the difference was in maternal psychological control $(U=354,000 ; p=.00)$. The maternal behavioral control was related to the disorder of disocial behavior ( $R h o=.222 ; p=.014 \leq .05$ ). Maternal psychological control and gender were related $(R h o=.240 ; p=.00 \leq .01)$.

Conclusions: The determining variable in the case of the sample of adolescents with antisocial behavior was maternal behavioral control and according to gender was the psychological control, confirming the importance of the influence of maternal practices in the construction of prosocial behavior in teenagers.

Keywords: Dissocial behavior, adolescent behavior, prosocial behavior, parental responsibility.

\section{Resumo}

Objetivo: verificar a relação entre práticas parentais e comportamentos anti-sociais em adolescentes vinculados ao sistema de responsabilidade criminal de adolescentes.

Método: o presente estudo enquadrou-se em uma investigação quantitativa transversal, descritiva-correlacional. A população foi composta por adolescentes vinculados ao sistema de responsabilidade criminal e 
Germán Cabrera Gutiérrez, Nora Helena Londoño Arredondo, Joan Sebastián Arbeláez Caro, María Camila Alvarez Martínez, Andrés Mauricio Gamboa Hernández

a amostra foi determinada a partir de amostragem não probabilística intencional. No total, participaram 70 adolescentes vinculados ao Sistema de Responsabilidade Criminal de Adolescentes (SRPA).

Instrumentos: ficha de caracterização, escala de práticas parentais de Andrade e Betancourt (2008) e entrevista neuropsiquiátrica para adolescentes (Ferrando et al., 2000).

Resultados: 80\% referem transtorno de comportamento anti-social. Verificou-se também que o controle comportamental materno apresenta diferença significativa em relação ao grupo de pessoas com transtorno antisocial $(U=1134.500 ; p=0,014)$ e, no caso do grupo gênero, a diferença foi no controle psicológico materno

$(U=354.000 ; p=0,00)$. 0 controle comportamental materno mostrou-se relacionado ao transtorno de conduta $(R h o=0,222 ; p=0,014 \leq 0,05)$. 0 controle psicológico materno e o gênero foram relacionados $(\mathrm{Rho}=0,240$; $p=0,00 \leq 0,01)$.

Conclusões: a variável determinante para o caso da amostra de adolescentes com comportamento anti-social foi o controle comportamental materno e quanto ao gênero foi o controle psicológico, confirmando a importância da influência das práticas maternas na construção do comportamento pró-social em adolescentes.

Palavras-chave: comportamento dissocial, comportamento do adolescente, pró-socialidade, responsabilidade parental.

\section{Introducción}

Para muchas disciplinas sociales y biológicas, la adolescencia es uno de los momentos del desarrollo con mayor número de implicaciones para el sujeto. La adolescencia supone una serie de metas y de conflictos a solucionar (García et al., 2020). Sin embargo, para el cumplimiento de dichas metas, el individuo dispone de las herramientas adquiridas en la niñez mediante los modelos que tuvo durante su aprendizaje primario. En ese sentido, Sánchez-Gómez et al. (2020) concluyen que las metas que suponen una alta dificultad para el sujeto derivan de las dimensiones biológicas y sociales que son explícitas en esta etapa del desarrollo.

En primer lugar, la propia definición del concepto de adolescencia es altamente complejo, ya que debe referir a un análisis sociológico y antropológico también. Paralelo al desarrollo del término adolescencia en el interior de la psicología, algunos análisis antropológicos, como el caso de Feixa (2011) o el propio Borrás (2014), han concluido que el concepto no es transversal a la mayoría de las culturas y se debe comprender a la luz de los fenómenos sociales que se traducen en metas para las personas que atraviesan la pubertad. La conclusión de algunos tratados en antropología y sociología sugieren que no es posible indicar fenómenos o características comunes en la adolescencia, y que es preciso advertir que cada cultura propone una serie de metas específicas en este estadio del desarrollo (Borrás, 2014; Feixa, 2011; García y Parada, 2018; Mendoza, 2018). Es así como, "pensar sociológicamente a los niños y los adolescentes implica comprender su lugar en el seno de las diferentes 
configuraciones de relaciones de interdependencia entre los actores que componen el universo familiar, el grupo de pares y la institución escolar" (Lahire, 2007, p. 23).

Por su parte, la psicología entiende al sujeto a tenor de su dimensión social, sumando, además, los factores culturales que determinan las metas que debe desarrollar el adolescente tal como apunta Díaz (2006). El adolescente es un sujeto que está atravesando por la pubertad y que justo en ese cambio biológico sucede la terminación de la construcción de su personalidad y de sus rasgos definitorio. Una excelente forma de explicar la adolescencia, la tiene Lozano (2014) cuando afirma: "la adolescencia se define como un periodo de transición en el estatus biosocial del individuo: el periodo que transcurre entre la madurez biológica y social" (p. 28).

En psicología, uno de los teóricos más interesado en el tema y que marcó un hito en la comprensión de la adolescencia fue Hall (Delval, 2002). Hall (1904) configuró un modelo teórico para comprender la adolescencia en función de los factores biológicos (pubertad) y los sociales (factores inter e intra relacionales). Hall (1904) parte del supuesto de que la adolescencia resulta ser un momento de tensión en el que se detona el desajuste biológico propio de la pubertad para demarcar el cambio biológico de independencia con su nicho primario. Sumado a esto, el sujeto debe hacerse de las herramientas previas que le fueron dotadas en la niñez para construir su rol de adulto y terminar de fundamentar los rasgos definitivos de su personalidad. El adolescente no solo hace uso de las herramientas aprehendidas en la niñez, sino que las diferentes etapas de la adolescencia le van permitiendo configurarse como un sujeto independiente en la medida que explora formas de relación, de acción y de pensamiento complejas, formales y con perspectivas de las que antes no podía ser capaz, tal y como concluye Meza (2017).

La familia, es el primer lugar de las relaciones inter e intraindividuales del sujeto en las que se delimitó el marco en que es posible el desarrollo de su personalidad. Según Mota (2014): "la individualidad se realiza, en el entorno familiar, por el parentesco, y genera una personalidad" (p. 284). La función de la familia antes de la pubertad y de las etapas de la adolescencia es la de formar las dimensiones cognitivas, emocionales y conductuales de la persona, gracias a la función relacional y de individualización. Esta conclusión coincide con lo analizado por Páez-Martínez (2017) acerca de las perspectivas más importantes de investigación sobre familia y adolescencia en Colombia.

En ese sentido, una de las metas o funciones de la adolescencia es la de construir identidad, para ello, hace uso de algunos elementos descritos por Ives (2014): "comunitario-social, historia, modelos psicohistóricos, modelos previos y conflicto" (p. 63). Cabe destacar, que los elementos descritos por Ives (2014) tienen su inicio 
Germán Cabrera Gutiérrez, Nora Helena Londoño Arredondo, Joan Sebastián Arbeláez Caro, María Camila Álvarez Martínez, Andrés Mauricio Gamboa Hernández 5

en la familia, ya que es en ella donde se identifican las funciones básicas culturales: identificación e individualización, además, es la familia el primer hito que sucede el conflicto para el adolescente, dado que la función de construir identidad lleva consigo el establecer independencia del modelo familiar.

Por otro lado, la familia es el enlace con las dimensiones comunitaria-social, ya que es por vía de sus características educativas y culturales, que el sujeto se adentra en las instituciones sociales por medio del lenguaje, representaciones simbólicas y construcción de sentido social. De allí que, para comprender al sujeto se requiere del conocimiento de sus dimensiones como el aspecto cognitivo, emocional-afectivo, conductual y relacional (Gaete, 2015; Velásquez, 2007).

La función de construcción de identidad implica la elaboración individual e independiente de los factores cognitivos, emocionales y conductuales. Para ello, el adolescente principalmente establece una forma de pensamiento más concreto, hipotético y lógico-causal (Ruiz, 2013). El procesamiento de información, que derivó de la estructuración de esquemas adaptativos propiciados en la familia, marcó la posibilidad de la evolución de dicho pensamiento en la adolescencia. El proceso de control autonoético y consciencia autonoética es fortalecido en la adolescencia, ya que es un efecto de la madurez biológica del córtex frontal (Cortés-Cortés et al., 2019). El proceso cognitivo es, sin lugar a duda, la primera dimensión en la comprensión del desarrollo del adolescente. Dicho aspecto se afecta según las características del desarrollo familiar y la formación de factores de riesgo (De Zubiría y De Zubiría, citados por Henao y Solorzano, 2012).

El aspecto cognitivo, por su lado, retroalimentado por factores o condiciones de riesgo para el adolescente, establece dinámicas adversas para el desarrollo normal, esto significa, no alcanzar los requerimientos o metas puntuales que supone la adolescencia: construir el rol de adulto y la fundamentación de los rasgos fundamentales de personalidad. Lo anterior, sucede porque, dado que existe la condición de riesgo, se generan afectaciones en la regulación emocional y conductas antisociales o comportamientos problemáticos en el adolescente. Las principales características de riesgo son descritas por Ruiz (2013): "circunstancias de la concepción y el embarazo, circunstancias perinatales adversas, características temperamentales difíciles, enfermedades crónicas, situaciones traumáticas concretas, características familiares" (p. 6).

La presente investigación plantea indagar sobre la relación entre los modelos de prácticas parentales en adolescentes en conflicto con la ley. El panorama de los adolescentes que han cometido conductas delictivas o antisociales es un tema de interés general, dado que es uno de los temas a tratar para la prevención y promoción 
de la salud mental y física de niños, niñas y adolescentes. En cifras, según datos del Instituto Colombiano de Bienestar Familiar (ICBF; 2018), para la fecha de corte junio 30 de 2018 eran 251455 menores que se encontraban en el Sistema de Responsabilidad Penal para Adolescentes (SRPA). En su mayoría, eran menores de 17 años (92 736 menores) seguidos de 16 años (76 991 menores).

Para el Ministerio de Salud y Protección Social (2016), la familia resulta ser un factor protector de las dinámicas de riesgo para el adolescente, sin embargo, también puede ser un factor de riesgo cuando la familia no se ha establecido adecuadamente o sus dinámicas interfieren en el desarrollo óptimo del sujeto. Dicha suposición, acerca de la familia y su influencia sobre las conductas de riesgo en el adolescente, nace de investigaciones previas que han establecido que hay una relación entre los factores familiares y la conducta de riesgo en el adolescente (Díaz-García y Moral-Jiménez, 2018; Paternina y Pereira, 2017).

Las prácticas de crianza han sido abordadas desde dos ópticas, la primera fue observarlas como un estilo específico de crianza, que el padre, madre o el cuidador tomaba con el menor. Dichos estilos se organizan a partir de las prácticas comunicativas y de establecimiento de normas. Otra óptica del constructo apunta a: "estudios más recientes consideran aspectos específicos de las prácticas parentales, donde se analizan el apoyo y el control de los padres e incluso se considera el efecto moderador de las características individuales de los hijos" (Andrade et al., 2012, p. 30).

Se ha advertido, líneas atrás, que la familia juega un papel significativo en la creación de la identidad del sujeto, fundamentalmente porque es el lugar donde se adquieren las primeras herramientas para los procesos de construcción de las dinámicas cognitivas, afectivas y conductuales de la persona, en particular. La familia impone dos rasgos en el sujeto: la comunicación y el tono afectivo (Martínez et al., 2019). La familia supone la introyección de los principales elementos que son importantes para elaborar el sentido de realidad del sujeto, además, es en ella que se gesta la regulación personal y la prosocialidad (Carrillo-Urrego, 2018; Pulido et al., 2013).

Kazdin y Buela-Casal (1994) describen, desde la evidencia empírica, que la familia es un factor de riesgo, al tiempo que protector en el inicio de la conducta antisocial. Puntualmente, Kazdin y Buela-Casal (1994) establecen que si bien la familia en su conjunto, es decir, todas las dimensiones que implica la familia están relacionados como factores de riesgo y de protección para la conducta antisocial, los estilos o prácticas que asumen los padres son altamente significativas para comprender la relación entre prácticas de crianza y conducta antisocial. Autores como Hirschi (1969) y Rodríguez et al. (2013), exploran el efecto de los estilos o prácticas de crianza que asumen los padres sobre la conducta antisocial, ellos coinciden en que el arraigo 
Germán Cabrera Gutiérrez, Nora Helena Londoño Arredondo, Joan Sebastián Arbeláez Caro, 7 María Camila Álvarez Martínez, Andrés Mauricio Gamboa Hernández

social y cultural, además las formas de relación con la norma social son aprendidas mediante las prácticas de crianza, pues ellas son modelos o esquemas que el menor introyecta acerca de cómo afrontar posteriormente la vida en común.

La evidencia disponible apunta, también, a que las prácticas de crianza moldean, como un factor social, las condiciones individuales, si se quiere estructurales, de la personalidad del adolescente (Gaeta y Galvanovskis, 2011; Marchiori, 2011). Aunque la personalidad en adolescentes no es del todo clara o visible como rasgos definitivos, la familia va moldeando dichos rasgos o corolarios individuales del sujeto. Por su parte, Rodríguez et al. (2013) y Moral-Jiménez y Pelayo-Pérez (2016) sintetizan que las prácticas de crianza hacen parte de la función de la familia sobre la aparición o formación de conductas antisociales. Por lo tanto, si la familia no cumple su función capacitadora de la norma social y, en cambio, introyecta en el adolescente un modelo de alteración de la norma social, podría ser un factor determinante para que el adolescente refiera la conducta disocial no deseada.

Así pues, si bien la familia está implicada en la configuración de conductas de riesgo para los adolescentes, la evidencia apunta a que la familia como un contexto, debe factorizarse en función de una mejor comprensión y, por tanto, una mejor delimitación de los modelos de investigación. La evidencia, como se ha descrito, apunta a que uno de los factores familiares significativos son las prácticas de crianza, igualmente, se ha puntualizado, grosso modo, del por qué los estilos que asumen los padres podrían llevar a que los adolescentes elicitan conductas antisociales o disociales.

El constructo de prácticas de crianza parental es elaborado por Andrade y Betancourt (2008), siguiendo tres elementos que las componen: comunicación, control conductual y psicológico y autonomía. Las prácticas que adoptan los padres funcionan como perpetuadores de modelos culturales y además son factores de riesgo, son amplificadores del contexto socioeconómicos que la contienen e influyen determinantemente en la elaboración de repertorios cognitivos y conductuales maladapatativos en los adolescentes (Cassidy, et al., 2013; Masten y Barnes, 2018).

Para delimitar mejor el constructo de prácticas de crianza parental, Andrade y Betancourt (2008) elaboran por cada padre una definición precisa siguiendo los elementos que la componen: 
Tabla 1. Dimensiones de la escala de prácticas parentales

\begin{tabular}{|c|c|c|}
\hline & Dimensión & Definición de la dimensión \\
\hline \multirow{4}{*}{ Papá } & $\begin{array}{l}\text { Comunicación y control } \\
\text { conductual paterno }\end{array}$ & $\begin{array}{l}\text { Comunicación existente entre el padre y el adolescente y conoci- } \\
\text { miento que tienen el padre de las actividades de sus hijos. }\end{array}$ \\
\hline & Autonomía paterna & $\begin{array}{l}\text { El respeto que muestra el padre en las decisiones que toman los } \\
\text { hijos. }\end{array}$ \\
\hline & Imposición paterna & El grado en que el padre impone sus creencias y conductas al hijo. \\
\hline & Control psicológico paterno & Inducción de culpa, devaluación y críticas excesivas al adolescente. \\
\hline \multirow{5}{*}{ Mamá } & Comunicación materna & Comunicación existente entre la madre y el adolescente. \\
\hline & Autonomía materna & $\begin{array}{l}\text { El respeto que muestran la madre en las decisiones que toman los } \\
\text { hijos. }\end{array}$ \\
\hline & Imposición materna & El grado en que la madre impone sus creencias y conductas al hijo. \\
\hline & Control psicológico materno & Inducción de culpa, devaluación y críticas excesivas al adolescente. \\
\hline & Control conductual Materno & Conocimiento que tienen la madre y el hijo sobre sus actividades. \\
\hline
\end{tabular}

Fuente: tomado de Andrade y Betancourt (2008, p. 12).

Andrade y Betancourt (2008), al precisar los factores de las prácticas de crianza, han demostrado que es una variable válida para su estudio, tal como se evidencia en diferentes investigaciones (Andrade, 2012; Méndez, 2013; Palacios y Andrade, 2008; Rodríguez y Mazón, 2020; Ruvalcaba-Romero et al., 2015).

Una vez se ha dejado claro que el presente estudio desea determinar la relación entre las prácticas de crianza parental y la conducta antisocial en adolescentes vinculados al sistema de responsabilidad penal para adolescente, se pretende, por lo tanto, describir, identificar diferencias y correlacionar las prácticas de crianzas en padres y madres prevalentes en adolescentes con y sin conducta antisocial.

\section{Metodología}

\section{Diseño}

Para comprender la relación entre las prácticas de crianza parental y la conducta antisocial en adolescentes en conflicto con la ley, el presente ejercicio de investigación siguió un enfoque cuantitativo, de tipo descriptivo con alcance correlacional en un marco de investigación transversal. Este modelo es preciso en las investigaciones no-experimentales, ya que pretendió entender no solo el comportamiento de las prácticas parentales según el instrumento que aquí se planteó en la población objeto de la investigación, sino que también entendió la relación y asociación entre las variables de estudio (Ato, López y Benavente, 2013). 
Germán Cabrera Gutiérrez, Nora Helena Londoño Arredondo, Joan Sebastián Arbeláez Caro, María Camila Álvarez Martínez, Andrés Mauricio Gamboa Hernández

\section{Participantes}

La población de la investigación fueron los adolescentes vinculados al sistema de responsabilidad penal para adolescentes (SRPA). Para que se proceda con la vinculación al SRPA, el adolescente ha cometido una infracción o conducta antisocial y delictiva y, por ello, se le sanciona. Sin embargo, su sanción es supervisada por el ICBF en un sistema que tiene por objeto la resocialización del menor y la protección de sus derechos. La muestra fue recogida siguiendo un método intencional no probabilístico, del que se obtuvieron, después de determinar los criterios de inclusión y de exclusión, un total de 70 adolescentes. Los criterios de inclusión fueron los siguientes: 1) que los sujetos se encontraran entre los 14 y 18 años de edad. 2) Contar con la firma de consentimiento informado del padre o madre y del adolescente. Por otro lado, los criterios de exclusión fueron: 1) que los participantes no presentaran trastornos mentales de base ni deterioro cognitivo. 2) Que no estuvieran bajo el efecto de alguna sustancia psicoactiva.

De los adolescentes encuestados, el 90\% corresponde a hombres y el restante 10\% a mujeres. La media de edad está en 16,3 años (DE=0,87), por su parte la media del estrato socioeconómico corresponde a 1,73 puntos ( $D E=0,79)$. En su mayoría, los adolescentes provienen de Armenia (44,3\%), seguido de Montenegro (14,3\%) a continuación Calarcá y La Tebaida (12,9\% respectivamente).

El 40\% de los adolescentes estudiaban antes de estar vinculados al SRPA, el $21,4 \%$ trabajaban y el 38,6\% ni trabajaba ni estudiaba. En promedio, el último grado escolar alcanzado por los adolescentes encuestados es 4 de primaria, evidenciando un bajo nivel académico. Los delitos de mayor prevalencia son hurto $(27,1 \%)$ y tráfico de estupefacientes (27,1\%), seguido de tentativa de homicidio (12,9\%), violencia intrafamiliar (8.6\%), homicidio y porte de armas (5,7\% respectivamente). En promedio, los adolescentes han ingresado una sola vez al SRPA. El 22,9\% de las familias de origen de los adolescentes es tradicional o nuclear (38,6\%), seguida de la familia monoparental femenina (20\%) y monoparental masculina $(11,4 \%)$.

\section{Instrumentos}

El primer instrumento aplicado fue la ficha de datos sociodemográficos. Aquí se indagó por las características sociales y familiares principales, así como las características del delito y el tiempo de vinculación al SRPA.

El segundo instrumento aplicado fue el de Prácticas parentales para adolescentes (EPP-A), es un instrumento realizado por Andrade y Betancourt (2008), que tiene por objeto evaluar la percepción que el adolescente tiene acerca de las prácticas de 
la crianza de sus padres. discriminando por papá y por mamá. Andrade y Betancourt (2008), orientaron cada uno de los ítems que componen esta escala tipo Likert a partir de las dimensiones de autonomía, comunicación, control conductual y control psicológico. La escala está compuesta por 40 reactivos sobre la madre y otros 40 reactivos sobre el padre. Existen cuatro posibilidades de respuesta: "nunca", "pocas veces", "muchas veces" y "siempre. La escala de fiabilidad utilizada por los autores fue el alfa de Cronbach, en dicho estudio osciló entre $a=0,80$ y $a=0,90$ (Andrade y Betancourt, 2008). En el presente estudio, la escala presentó un alfa de Cronbach que osciló entre $a=0,72$ y $a=0,85$.

El tercer instrumento fue La Entrevista neuropsiquiátrica para niños y adolescentes (M.I.N.I KID) en su versión en español de Colón-Soto et al. (1992). Se aplicaron de manera individual los ítems $L$ (dependencia y abuso de alcohol), el cual consta de 12 preguntas cerradas con posibilidad de respuesta dicotómica (Sí/No); la M (dependencia y abuso de sustancias) que consta de 13 preguntas; la P (trastornos de la conducta disocial) que tiene de 16 preguntas y $\mathrm{R}$ (trastorno psicótico) con un total de 20 preguntas. Se usaron, específicamente, los ítems de la dimensión L y M, referidos al consumo de alcohol y sustancias psicoactivas, en función de los hallazgos en los antecedentes, que indican que el consumo de sustancias está implicado en la conducta antisocial como un factor explicativo de la misma. El factor P se usó como la variable dependiente del estudio, ya que fue el filtro para encontrar si existe conducta disocial en la muestra de adolescentes encuestados. El factor $\mathrm{R}$ se usó en función de los criterios de exclusión, ya que adolescentes con trastorno psicótico eran reportados a la institución y no podrían pertenecer al estudio.

\section{Procedimiento}

El primer paso para la investigación fue la delimitación teórica y elección de los instrumentos de estudio. Acto seguido, se procedió a buscar el convenio interinstitucional entre la Universidad de San Buenaventura y el ICBF, que se logró mediante el Convenio de cooperación interinstitucional No. 63215 - 2017. A continuación, se envió el consentimiento informado al comité de bioética de la Universidad de San Buenaventura. Con su aprobación se llevó a cabo el trabajo de campo con las familias de los adolescentes para indicarles los riesgos, métodos e instrumentos de la investigación, con lo que se inició la aplicación en los adolescentes. 


\section{Estrategia analítica}

Con los datos obtenidos en el trabajo de campo, se realizó el análisis estadístico en el programa SPSS (Versión 23). La base con los datos se analizó siguiendo las orientaciones de la estadística descriptiva e inferencial. El primer procedimiento de los datos fue realizar un análisis de las medidas de tendencia central y de frecuencia según correspondía, de acuerdo con la naturaleza de las variables. A continuación, se realizó una prueba de hipótesis de normalidad, para identificar si se usaba estadísticas paramétrica o no paramétrica. Se concluyó, con un nivel de confianza del 95\%, que se debía usar estadística no paramétrica por la organización anormal de las variables. Así, para el caso de las comparaciones entre los grupos de adolescentes con y sin conducta antisocial, se usó el estadístico de la $U$ de Mann Whitney, para el caso de las correlaciones se usó el Rho de Spearman, ambas siguieron igualmente un nivel de 95\% de confianza para determinar la significación estadística.

\section{Resultados}

\section{Descriptivos de las variables de estudio}

Acerca de los datos recopilados mediante la entrevista neuropsiquiátrica, se encontró que el 23,9\% refiere abuso de bebidas alcohólicas, por otro lado, un 11,4\% no consume ninguna sustancia psicoactiva, de frente a 50\% que consume marihuana, 22,9\% cocaína, $8,6 \%$ estimulantes, $5,7 \%$ bazuco y un $1,4 \%$ medicinas psiquiátricas sin prescripción. El 71,4\% refieren dependencia a la sustancia psicoactiva de consumo. De acuerdo con el MINIKID, el 80\% de los adolescentes presenta trastorno de conducta antisocial y el 30\% refiere trastorno psicótico actual.

Respecto a los datos recopilados mediante la escala de prácticas parentales, se encontró que existe una alta variabilidad o dispersión, debido a que, en su mayoría, los adolescentes no tienen contacto con el padre, lo que hace, para el caso del análisis del padre, que la escala presente una alta dispersión de los datos (figura 2). Para el caso de la madre, la más alta puntuación se presentó en la dimensión de comunicación materna, la de menor puntuación se observó en el control psicológico materno (figura 1). Un elemento interesante, tiene que ver con la tendencia presentada en el diagrama de caja respecto a la tendencia estadística de la muestra a tener puntuaciones altas en las dimensiones de la madre (figura 1) y tener puntuaciones bajas para el caso del padre (figura 2). El dato más homogéneo se presentó en la dimensión de control conductual materno. 


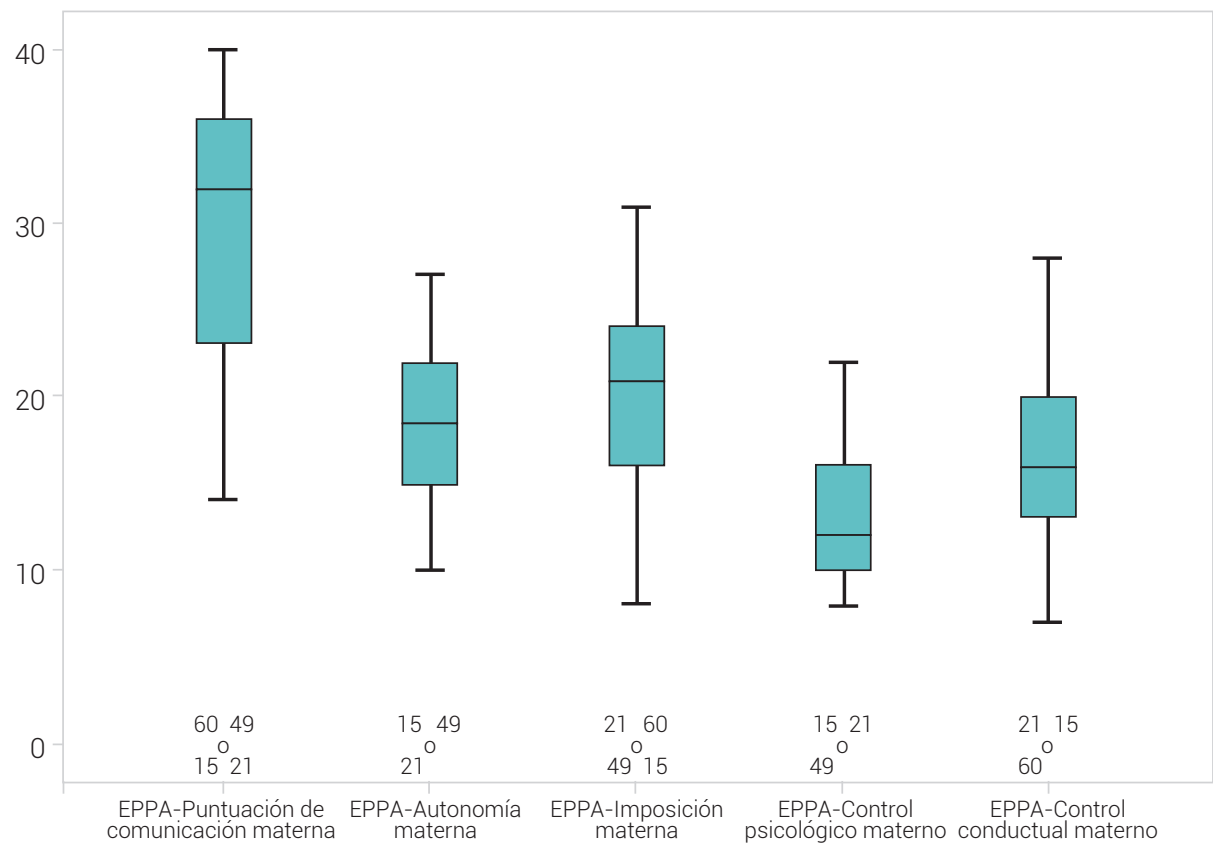

Figura 1. Medidas de tendencia central para las dimensiones de la madre EPP-A Fuente: elaboración propia.

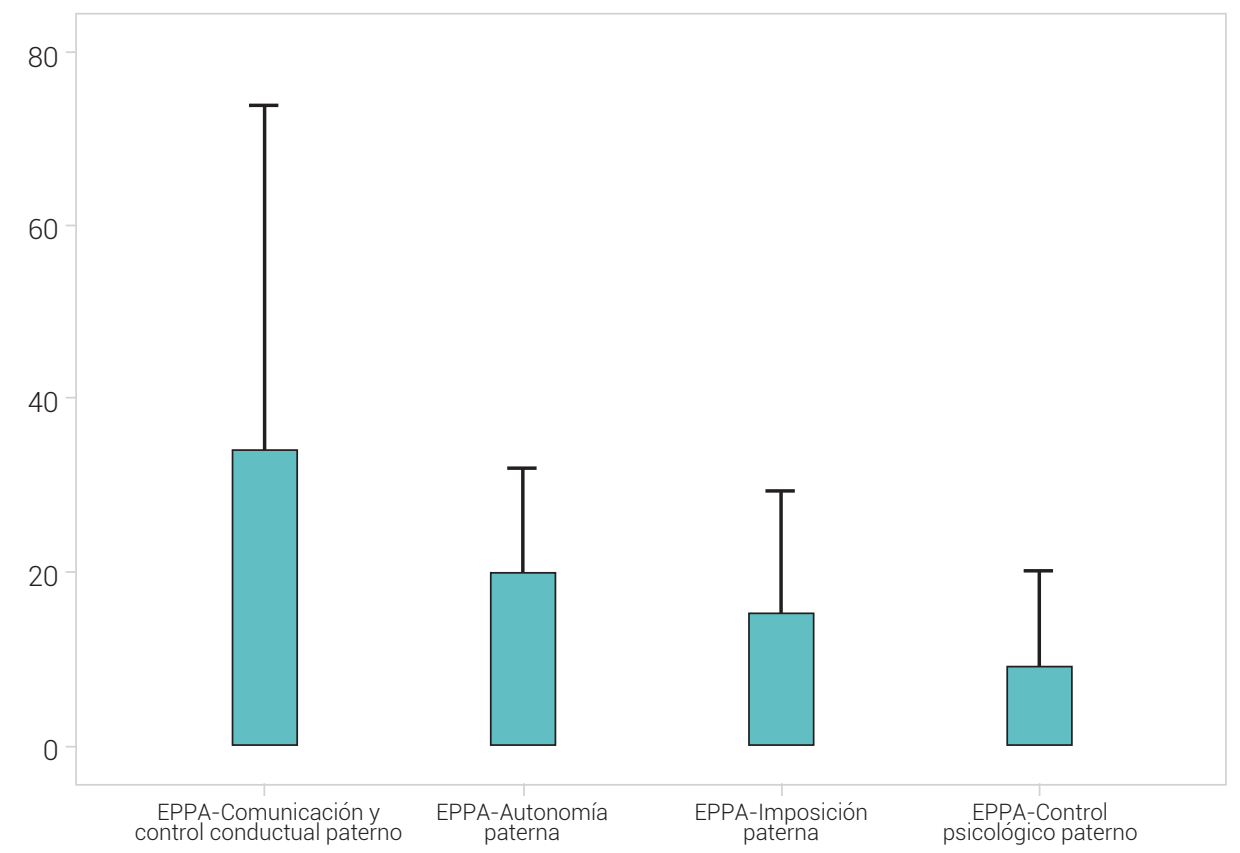

Figura 2. Medidas de tendencia central de las dimensiones del padre de la EPP-A. Fuente: elaboración propia. 


\section{Comparación entre grupos}

Los datos recopilados mediante la escala fueron estudiados según las diferencias de grupos. Puntualmente, en los que algunas variables demostraron diferencias, fue en los grupos de género y en los grupos de trastorno de conducta disocial y no trastorno de conducta disocial (tabla 2). En el caso de control conductual materno, se notó una diferencia significativa respecto al grupo de las personas con trastorno antisocial $(U=1134,500 ; p=0,014)$ y, en el caso del grupo de género, la diferencia estaba en el control psicológico materno $(U=354,000 ; p=0,00)$ (tabla 2).

Tabla 2. Comparativo de la escala de prácticas de crianza según género y trastorno disocial

\begin{tabular}{|c|c|c|c|c|c|c|c|}
\hline \multirow{3}{*}{ Indicador } & \multirow{2}{*}{\multicolumn{2}{|c|}{$\begin{array}{c}\begin{array}{c}\text { Trastorno de } \\
\text { conducta }\end{array} \\
N=56\end{array}$}} & \multirow{2}{*}{\multicolumn{2}{|c|}{$\begin{array}{l}\text { No trastorno } \\
\text { de conducta }\end{array}$}} & \multirow{3}{*}{$\begin{array}{l}\text { U de Mann- } \\
\text { Whitney }\end{array}$} & \multirow{3}{*}{$p$} & \multirow{3}{*}{$d$. } \\
\hline & & & & & & & \\
\hline & M & $D t$ & M & $D t$ & & & \\
\hline EPPA-Comunicación materna & 29 & 11 & 30 & 7 & 1521.5 & 0,776 & $(-) 0,28$ \\
\hline EPPA-Autonomía materna & 18 & 7 & 18 & 4 & 1532 & 0,821 & $(-) 0,22$ \\
\hline EPPA-Imposición materna & 18 & 8 & 21 & 4 & 1480.5 & 0,608 & $(-) 0,513$ \\
\hline EPPA-Control psicológico materno & 12 & 5 & 16 & 4 & 1522 & 0,778 & $(-) 0,28$ \\
\hline EPPA-Control conductual materno & 16 & 7 & 18 & 5 & 1134.5 & 0,014 & $(-) 2,44$ \\
\hline $\begin{array}{l}\text { EPPA-Comunicación y control con- } \\
\text { ductual paterno }\end{array}$ & 18 & 23 & 15 & 23 & 1546.5 & 0,871 & $(-) 0,16$ \\
\hline EPPA-Autonomía paterna & 10 & 12 & 7 & 11 & 1567.5 & 0,975 & $(-) 0,03$ \\
\hline EPPA-Imposición paterna & 8 & 10 & 7 & 11 & 1547.5 & 0,876 & $(-) 0,15$ \\
\hline \multirow[t]{3}{*}{ EPPA-Control psicológico paterno } & 5 & 7 & 4 & 6 & 1527.5 & 0,778 & $(-) 0,28$ \\
\hline & \multicolumn{2}{|c|}{ Hombre } & \multicolumn{2}{|c|}{ Mujer } & \multirow{2}{*}{$\begin{array}{l}\text { U de Mann- } \\
\text { Whitney }\end{array}$} & \multirow{2}{*}{$p$} & \multirow{2}{*}{$d$} \\
\hline & \multicolumn{2}{|c|}{$N=63$} & \multicolumn{2}{|c|}{$N=7$} & & & \\
\hline EPPA-Comunicación materna & 29 & 10 & 29 & 11 & 638,000 & 0,850 & $(-) 0,189$ \\
\hline EPPA-Autonomía materna & 18 & 7 & 18 & 7 & 596,500 & 0,584 & $(-) 0,548$ \\
\hline EPPA-Imposición materna & 19 & 7 & 18 & 7 & 543,500 & 0,316 & $(-) 1,00$ \\
\hline EPPA-Control psicológico materno & 12 & 5 & 12 & 5 & 354,000 & 0,008 & $(-) 2,63$ \\
\hline EPPA-Control conductual materno & 15 & 6 & 18 & 7 & 534,000 & 0,278 & $(-) 1,08$ \\
\hline $\begin{array}{l}\text { EPPA-Comunicación y control con- } \\
\text { ductual paterno }\end{array}$ & 18 & 23 & 18 & 24 & 614,000 & 0,657 & $(-) 0,44$ \\
\hline EPPA-Autonomía paterna & 9 & 12 & 9 & 12 & 591,500 & 0,509 & $(-) 0,66$ \\
\hline EPPA-Imposición paterna & 8 & 10 & 7 & 10 & 641,500 & 0,858 & $(-) 0,17$ \\
\hline EPPA-Control psicológico paterno & 5 & 7 & 5 & 6 & 621,000 & 0,707 & $(-) 0,37$ \\
\hline
\end{tabular}

Nota. M: media; DT: Desviación típica; p: significación; d: efecto de la muestra.

Fuente: elaboración propia. 


\section{Correlación entre variables}

Por último, se realizó un análisis de correlación entre las variables de estudio, se encontró que sólo el control conductual materno tenía correlación con el trastorno de conducta disocial (Rho=0,222; $p=0,014 \leq 0,05)$. Otra correlación evidenciada fue la de control psicológico materno y el género ( $R$ ho = 0,240; $p=0,00 \leq 0,01)$.

\section{Discusión}

El presente estudio tenía por objeto principal el de describir, identificar diferencias y correlacionar las prácticas de crianzas en padres y madres prevalentes en adolescentes con y sin conducta antisocial, vinculados al sistema de responsabilidad penal para adolescentes. Se encontró una relación específica entre el comportamiento antisocial y el control conductual materno, además de su diferencia entre grupos. Resulta interesante el comportamiento específico del efecto del control conductual como una variable determinante sobre la conducta antisocial y delictiva en los adolescentes.

En contexto, conviene afirmar la importancia de elaborar el constructo de la relación de padres con los hijos en función de las prácticas parentales y no como estilos de crianza, ya que el modelo seguido por este ejercicio de investigación tiene mejores características para el estudio de la influencia de la relación entre padres e hijos (Rivera y Cahuana, 2016). Las prácticas de crianza como constructo de investigación ha demostrado eficacia a nivel teórico (Casais, et al., 2017; Méndez, et al., 2013; Ruvalcaba-Romero, et al., 2015; Zacarías-Salinas y Andrade-Palos, 2014).

En la construcción de conductas prosociales del adolescente, la relación específica con cada uno de los cuidadores o padres es determinante para establecer las condiciones efectivas del comportamiento social y legal. El agenciamiento moral del sujeto, además de la formación de prácticas cognitivas y conductuales sanas, implica la relación con los padres, en tanto los roles de padre y de madre fungen como arquitectos de los rasgos particulares del adolescente. Se encontró, gracias a los resultados arrojados por la investigación, que el rol materno es esencial para la comprensión del comportamiento antisocial, quizá porque, en atención a investigación previas a la luz de la presente, es el rol de la madre la que propone límites conductuales a partir del tono afectivo y comunicativo (Palacios y Andrade, 2008).

La madre, en su rol de cuidadora, es una supervisora que, gradualmente, va proponiendo los aspectos particulares en el comportamiento prosocial (Peralta et al., 2018). Los propios autores de la escala de prácticas parentales afirman, respecto al control conductual de la madre, que se refiere a: "un conjunto de conductas parentales 
que involucran atención y supervisión de las actividades de los hijos, referido en varias investigaciones como supervisión o monitoreo" (Betancourt y Andrade, 2011, p. 28). El monitoreo conductual es la delimitación en términos lógicos-deductivos del comportamiento tendiente a la norma, ajuste positivo del otro y retroalimentación de la estructura cognitiva-afectiva-conductual del adolescente. El monitoreo requiere tanto del rol paterno como del materno, sin embargo, aquí no se encontró una relación para el padre, quizá por la alta prevalencia de familias monoparentales femeninas, fenómeno interesante de entender a la luz del comportamiento antisocial del adolescente, pues, si el control conductual materno es una variable implicada en la formación de conducta antisocial, se podría prevenir en virtud de dichos resultados y puede ser útil para la intervención de la conducta antisocial. Estos datos también deberían ser interesantes dado que, en el departamento del Quindío, hay un notable crecimiento de las familias monoparentales femeninas (Secretaría de Salud Departamental, 2018).

También, si existen prácticas de crianza parental inadecuadas en la relación materno-filial, se puede formar con contexto de vulnerabilidad que puede incidir directamente en la estructuración de desregulación afectiva y esto, a su vez, puede influir sobre comportamiento antisocial (Hawkins y Haskett, 2014; Romero, 2015; VargasRubilar, et al., 2018). En la investigación, la regulación conductual del comportamiento ha demostrado una relación con la regulación emocional (Ruvalcaba-Romero et al., 2018), por lo tanto, es importante para posteriores investigaciones indagar sobre dicha relación específica.

Paralelo a lo anterior, otra característica diferenciadora del estudio fue el género en función del control psicológico de la madre. Investigaciones previas, han demostrado que, para la comprensión de la conducta antisocial, se precisa identificar las diferencias por género, dado que el Ilamado "acople" a la norma no funciona de igual manera para ambos géneros (Sánchez et al., 2018; Rodríguez y Duque, 2011). El control psicológico, por su parte, tiene que ver con el monitoreo de las dimensiones cognitivas-afectivas-comportamentales de la conducta prosocial, allí la madre, tal como se evidenció, es esencial para prevenir o para influir sobre conductas antisociales según hombres y mujeres. En ese sentido, se confirman investigaciones previas que han indagado sobre este último punto, comprobando que el control psicológico y el modelo en sí mismo de la madre, es diferente para hombres y para mujeres (Shehzadi et al., 2018). La madre no tiene un rol concreto o establecido, no debería comprenderse que el rol asumido mediante las prácticas de crianza son un esquema o rol esperado de la madre en la familia, antes bien, cada rol asumido por padre, madre o cuidador varía, según las necesidades del contexto, de la familia y del menor mismo. 
Lo anterior debe comprenderse, también, a la luz de los datos que recopila el ICBF (2018), sobre la dinámica de la conducta antisocial, ya que es más prevalente en hombres que en mujeres. Siguiendo este punto, investigaciones futuras deben inquirir sobre la relación individual de padre y madre y su relación diferencial entre hombres y mujeres, partiendo de la más alta prevalencia de la conducta delictiva en hombres que en mujeres. Autores como Calleja et al. (2018), encontraron que el factor psicológico en las prácticas parentales en un factor predictivo acerca de la aparición de conductas antisociales.

En suma, la conducta antisocial en adolescentes es un fenómeno que por sí mismo es interesante en el contexto de las ciencias sociales, sin embargo, su precisión en términos de investigación debe comprenderse como un factor de riesgo o como un efecto de ciertas variables, aquí se exploró la influencia de las prácticas de crianza parental y se encontró la importancia del control tanto psicológico como conductual por parte de la madre (Ekechukwu, 2018).

Dos fenómenos interesantes también se observaron en el estudio, el primero tiene que ver con la alta prevalencia de consumo de sustancias psicoactivas y el segundo es que no todos los adolescentes que están vinculados al SRPA presentan trastorno disocial.

Por un lado, el consumo de sustancias psicoactivas se ha definido como un factor de riesgo de frente a la aparición de conducta antisocial, así lo han concluido algunos estudios actuales (Rodríguez, 2016; Sanabria y Uribe, 2010; Sánchez et al., 2017). Por tanto, dada una mayor prevalencia de consumo de sustancias psicoactivas, se puede entender que exista una mayor proclividad a la comisión de conductas antisociales. Puntualmente, el efecto del consumo de sustancias psicoactivas sobre la conducta antisocial, se entiende porque está en el contexto de las conductas de riesgo del adolescente. Estas, a su vez, expresan la desregulación cognitiva-conductual propia de la etapa de la pubertad y de las etapas primarias de la adolescencia, allí las sustancias psicoactivas funcionan en muchos casos como amalgamadores sociales para con los grupos que el adolescente explora, además, las sustancias no solo tienen un efecto social, sino un deterioro de las dimensiones individuales del sujeto.

Por otro lado, no todos los adolescentes vinculados al SRPA presentan trastorno disocial, ya que si bien hay una relación previamente demostrada sobre el trastorno disocial y la conducta antisocial (Padrón y García, 2018), existen tipos de delitos que por sí mismo, no hacen parte de una conducta antisocial, sino que por su naturaleza responden a otras intenciones o circunstancias. Para futuras investigaciones o reflexiones sobre el tema, debe ahondarse, en primer lugar, sobre este respecto, pues no es claro hasta qué punto, el estar en el sistema de responsabilidad penal o, 
posteriormente, en la cárcel, implicaría conducta antisocial o un trastorno de conducta antisocial. No obstante, el estar vinculados al sistema de responsabilidad penal, indica que hay una proclividad a tener conductas antisociales tal como se evidenció en el estudio, sumando a ello que los adolescentes vinculados comporten factores de riesgo, principalmente, el consumo de sustancias psicoactivas.

\section{Limitaciones y conclusiones}

La limitación principal del estudio tiene que ver con el acceso a la muestra, ya que si bien se contó con la aprobación de las instituciones encargadas del cuidado de los adolescentes del SRPA, las familias de estos no son asiduas en las visitas y la evolución de sus hijos, en ese sentido, esta limitación es un hallazgo de la investigación, ya que hay muy poca vinculación de las familias al proceso de resocialización de los adolescentes en conflicto con la ley. Dados los resultados de la investigación, se encontró que la importancia del vínculo materno en función del control psicológico y conductual, se sugiere que en futuras investigaciones se indague sobre el efecto de la desvinculación de las familias en el proceso de resocialización de los adolescentes en conflicto con la ley.

En conclusión, el estudio encontró que las prácticas parentales influyen en la comprensión de la conducta antisocial de adolescentes, puntualmente se asoció el control conductual con la aparición de conducta antisocial y delictiva y que, en función del género, el control psicológico tiene una influencia importante. Por otro lado, se encontró una alta prevalencia de consumo de sustancias psicoactivas que funge como factor de riesgo en los adolescentes para la comisión de conductas antisociales. Se encontró también que no todos los adolescentes vinculados al SRPA tienen trastorno disocial. Se sugiere para futuras investigaciones indagar la relación diferenciadora del padre y de la madre y su influencia predictora en la aparición de comportamiento delictivo.

\section{Conflicto de intereses}

Los autores declaran ningún conflicto de interés. 


\section{Referencias}

Andrade, P. P. y Betancourt, O. D. (2008). Escala Prácticas Parentales para adolescentes: PP-A. Universidad Nacional Autónoma de México.

Andrade, P., Betancourt, O., Vallejo, A., Celis, B. y Rojas, R. (2012). Prácticas parentales y sintomatología depresiva en adolescentes. Salud mental, 35, 29-36.

Ato. M., López., J. y Benavente, A. (2013). Un sistema de clasificación de los diseños de investigación en psicología. Anales de psicología, 29(3), 1038-1059. http://dx.doi.org/10.6018/ analesps.29.3.178511

Betancourt, D. y Andrade, P. (2011). Control parental y problemas emocionales y de conducta en adolescentes. Revista Colombiana de Psicología, 20(1), 27-41.

Borrás, T. (2014). Adolescencia: definición. vulnerabilidad y oportunidad. Correo científico medico de Holguín, 18(1), 5-7.

Calleja, N., Álvarez, A., Contreras, R. y Nares, P. (2018). Estilos de crianza como predictores del comportamiento tabáquico adolescente. Pensamiento psicológico, 16(1), 7-18. https://doi. org/10.11144/Javerianacali.PPSI16-1.ecpc

Carrillo-Urrego, A. (2018). Castigos en la crianza de los hijos e hijas: un estado de la cuestión. Revista Latinoamericana de Ciencias Sociales, Niñez y Juventud, 16(2), 719-740. https://doi. $\operatorname{org} / 10.11600 / 1692715 \times .16206$

Casais, D., Flores, M. y Domínguez, A. (2017). Percepción de prácticas de crianza: análisis confirmatorio de una escala para adolescentes. Acta de investigación psicológica, 7(2), 2717-2726. https://doi.org/10.1016/j.aipprr.2017.06.001

Cassidy. J., Jones, J. \& Shaver, P. (2013). Contributions of Attachment Theory and Research: A Framework for Future Research. Translation. and Policy. Development and Psychopathology, 25(4), 1415-1434. https://doi.org/10.1017/S0954579413000692

Colón-Soto, M., Díaz, V., Soto, O. \& Santana, C. (2005). Mini International Neuropsychiatric Interview para Niños y Adolescentes (MINI-KID) Versión en español. Medical Outcome Systems.

Cortés-Cortés, M., Alfaro, A., Martínez, V. y Veloso, B. (2019). Desarrollo cerebral y aprendizaje en adolescentes: Importancia de la actividad física. Revista médica de Chile, 147, 130-131. 
Delval, J. (2002). El desarrollo humano. Siglo XXI.

Díaz, J. (2006). Identidad, adolescencia y cultura. Jóvenes secundarios en un contexto regional. Revista mexicana de investigación educativa, 11(29), 431-457.

Díaz-García, N. y Moral-Jiménez, M. V. (2018). Consumo de alcohol y conducta antisocial e impulsividad en adolescentes españoles. Acta colombiana de Psicología, 21(2), 110-120. http://www. dx.doi.org/10.14718/ACP.2018.21.2.6

Ekechukwu, R. (2018). Influence of Family Type (Single/Step-Parent) and Parenting Styles (Authoritarian/Authoritative) on Adolescent's Social Behaviour in Yenagoa L.G.A. of Bayelsa State. International Journal of Humanities Social Sciences and Education, 5(1), 92-97. http:// dx.doi.org/10.20431/2349-0381.0501014

Feixa, C. (2011). Past and Present of Adolescence in Society. The ‘Teen Brain’ Debate in Perspective. Neuroscience and Biobehavioral Reviews, 35(8).

Ferrando, L., Bobes, J. \& Gibert, J. (2000). Mini International Neuropsychiatric Interview. Instituto IAP-Madrid.

Gaeta, M. y Galvanovskis, A. (2011). Propensión a conductas antisociales y delictivas en adolescentes mexicanos. Psicología Iberoamericana,19(2),47- 54.

Gaete, V. (2015). Desarrollo psicosocial del adolescente. Revista chilena de pediatría, 86(6), 436-443. https://doi.org/10.1016/j.rchipe.2015.07.005

García, C. y Parada, D. (2018). “Construcción de adolescencia”: una concepción histórica y social inserta en las políticas públicas. Universitas Humanistica, 85, 347-373. https://doi.org/10.11144/ Javeriana.uh85.cach

García, D., Hernández-Lalinde, J., Espinosa-Castro, J-F. y Soler, M. (2020). Salud mental en la adolescencia montevideana: una mirada desde el bienestar psicológico. Archivos Venezolanos de Farmacología y Terapéutica, 39(2), 182-190. https://bit.ly/3vToHCU

Hall, S. (1904). Adolescence. Appleton

Hawkins, A. \& Haskett, M. (2014). Internal Working Models and Adjustment of Physically Abused Children: The Mediating Role of Self-Regulatory Abilities. The Journal of Child Psychology and Psychiatry, 55(2), 135-143. https://doi.org/10.1111/jcpp.12118

Henao, M. y Solórzano, B. (2012). Una aproximación al desarrollo del pensamiento en el adolescente. Revista universitaria Eafit, 100, 53-60. 
Hirschi, T. (1969). Causes of Delinquency. Universidad de California.

Instituto Colombiano de Bienestar Familiar. (ICBF). (2018). Política nacional de infancia y adolescencia 2018-2030. https://bit.ly/3iRFa5o

Ives, E. (2014). La identidad del Adolescente. Como se construye. Adolescere, 2(2), 14-18.

Kazdin, A. y Buela-Casal, A. (1994). Conducta Antisocial. Evaluación, tratamiento y prevención en la infancia y adolescencia. Pirámide.

Lahire, B. (2007). Infancia y adolescencia: de los tiempos de socialización sometidos a constricciones múltiples. Revista de antropología social, 16, 21-38.

Lozano, A. (2014). Teoría de teorías sobre la adolescencia. Última década, 40, 11-36.

Marchiori, H. (2011). Psicología criminal. Porrua,

Martínez, D., Amaya, D. y Calle, P. A. (2019). Prácticas de crianza y comunicación familiar: una estrategia para la socialización primaria. Poiésis, 16(36), 111-125. https://doi.org/10.2150 $1 / 16920945.3193$

Masten, A. S. \& Barnes, A. J. (2018). Resilience in Children: Developmental Perspectives. Children, 5(7), 98. https://doi.org/10.3390/children5070098

Méndez, M., Andrade, P. y Peñaloza, R. (2013). Prácticas parentales y capacidades y dificultades en preadolescentes. Revista Intercontinental de Psicología y Educación, 15(1), 99-118.

Mendoza, R. (2018). La adolescencia como fenómeno cultural. Universidad de Huelva.

Meza, I. (2017). Ezequiel A. Chávez y la secularización del adolescente mexicano: Ensayo de psicología de la adolescencia (1928). Relaciones Estudios de Historia y Sociedad. 150, 247-279. https://doi.org/10.24901/rehs.v38i150.303

Ministerio de Salud y Protección Social. (2016). Boletín de salud mental Salud mental en niños. niñas y adolescentes. https://bit.ly/3mD2Z1y

Mota, A. (2014). Una reflexión hermenéutico-analógica del parentesco como carácter simbólico de la conformación de las relaciones humanas en la forma de familia. Efemérides mexicanas, 32(95), 264-285. 
Moral-Jiménez, M. \& Pelayo-Pérez, L. (2016). Factores sociodemográficos y familiares en menores de España con medida judicial, cívicos e infractores. Revista Latinoamericana de Ciencias Sociales, Niñezy Juventud, 14(2), 1217-1233. https://doi.org/10.11600/1692715x.14223210715

Padrón, C. y García, T. (2018). Trastornos mentales y homicidio. Revista Cubana de Medicina General Integral, 34(1), 4-13.

Páez-Martínez, R. (2017). Tendencias de investigaciones sobre la familia en Colombia. Una perspectiva educativa. Revista Latinoamericana de Ciencias Sociales, Niñezy Juventud, 15(2), 823837. https://doi.org/10.11600/1692715x.1520331052016

Palacios, J. y Andrade, P. (2008). Influencia de las prácticas parentales en las conductas problemas de los adolescentes. Investigación Universitaria Multidisciplinaria, 7(7), 7-18.

Paternina, D. y Pereira, M. (2017). Funcionalidad familiar en escolares con comportamientos de riesgo psicosocial en una institución educativa de Sincelejo (Colombia). Salud Uninorte, 33(3), 429-437.

Peralta, A., Therán, A., Pardo, M. y Sánchez, S. (2018). La responsabilidad parental en adolescentes infractores de la ley colombiana. alcances y limitaciones para la reducción de la criminalidad juvenil. [Trabajo de grado para optar al título de abogado]. Universidad Cooperativa de Colombia, Villavicencio, Facultad de Derecho.

Pulido, S., Castro-Osorio, J., Peña, M. y Ariza-Ramírez, D. P. (2013). Pautas, creencias y prácticas de crianza relacionadas con el castigo y su transmisión generacional. Revista Latinoamericana de Ciencias Sociales, Niñez y Juventud, 11(1), 245-259.

Rivera, R. y Cahuana, M. (2016). Influencia de la familia sobre las conductas antisociales en adolescentes de Arequipa-Perú. Actualidades en psicología, 30(120), 85-97. http://dx.doi. org/10.15517/ap.v30i120.18814

Rodríguez, H., Espinosa, A. y Pardo, C. (2013). Función familiar y conductas antisociales y delictivas de adolescentes de instituciones públicas educativas de la ciudad de Ibagué - Colombia. Revista vanguardia psicológica, 3(2), 137-149.

Rodríguez, J. y Duque, A. (2011). Factores de protección y conducta antisocial en adolescentes escolarizados: Un análisis de género. Capítulo criminológico, 39, 49-82.

Rodríguez, L. M. (2016). Conductas antisociales: entorno y autoestima en adolescentes mexicanos. Trabajo Social Hoy, 78, 7-27. http://dx.doi.org/10.12960/issn.1134-0991 
Rodríguez, P. y Mazón, J. (2020). Estilos parentales y acoso escolar en una muestra de niños de primaria. Alternativas en psicología, 44, 37-52.

Romero, N. (2015). Prácticas de crianza, ansiedad y regulación emocional en contexto escolar. [Trabajo de grado presentado; Maestría en Psicología]. Repositorio UNAL. https://bit. ly/3boakgk

Ruiz, L. (2013). Psicología del adolescente y su entorno. Siete días médicos. https://bit.ly/3arxbHx

Ruvalcaba-Romero, N., Gallegos-Guajardo, J., Caballo. V. y Villegas-Guinea, D. (2015). Prácticas parentales e indicadores de salud mental en adolescentes. Psicología desde el caribe, 33(3), 223-236. http://dx.doi.org/10.14482/psdc.33.3.9485

Sanabria, A. y Uribe, A. (2010). Factores psicosociales de riesgo asociados a conductas problemáticas en jóvenes infractores y no infractores. Perspectivas psicológicas, 6(2), 257-274.

Sánchez, A., Xóchitl, I. y Robles, F. (2018). Conductas antisociales-delictivas en adolescentes: relación con el género. la estructura familiar y el rendimiento académico. Alternativas psicológicas, 38, 80-98.

Sánchez-Gómez, M., Oliver, A., Adelantado-Renau, M., y Bresó, E. (2020). Inteligencia emocional y ansiedad en adolescentes: una propuesta práctica en el aula. Quaderns Digitals: Revista de Nuevas Tecnologías y Sociedad, (91), 74-89. https://bit.ly/3CE5Jm4

Secretaría de Salud Departamental del Quindío. (2018). Análisis de Situación en Salud.

Shehzadi, F., Safdar, R., Batool, I., \& Ali, A. (2018). Effect of Perceived Parenting Styles on Disruptive Behavior among Adolescents. Peshawar Journal of Psychology and Behavioral Sciences, 4(1), $1-13$.

Vargas-Rubilar, J. A., Richaud, M. C., y Oros, L. B. (2018). Programa de promoción de la parentalidad positiva en la escuela: un estudio preliminar en un contexto de vulnerabilidad social. Pensando Psicología, 14(23). https://doi.org/10.16925/pe.v14i23.2265

Velásquez, A. (2007). Lenguaje e identidad en los adolescentes de hoy. Ágora, 7(1), 1-197.

Zacarías-Salinas. X. y Andrade, P. (2014). Una escala para evaluar prácticas parentales que promueven la conducta prosocial en preadolescentes. Revista Iberoamericana de Diagnóstico y Evaluación, 2(38), 117-135. 